Archives de sciences sociales des religions

135 | juillet - septembre 2006

Réveils du soufisme en Afrique et en Asie

\title{
Mystique et politique chez Abdessalam Yassine et ses adeptes
}

Youssef Belal

\section{(2) OpenEdition}

1 Journals

Édition électronique

URL : http://journals.openedition.org/assr/3790

DOI : 10.4000/assr.3790

ISSN : $1777-5825$

Éditeur

Éditions de l'EHESS

Édition imprimée

Date de publication : 1 septembre 2006

Pagination : 165-184

ISBN : 2-7132-2093-9

ISSN : 0335-5985

\section{Référence électronique}

Youssef Belal, « Mystique et politique chez Abdessalam Yassine et ses adeptes », Archives de sciences sociales des religions [En ligne], 135 | juillet - septembre 2006, mis en ligne le 16 octobre 2009, consulté le 01 mai 2019. URL : http://journals.openedition.org/assr/3790 ; DOI : 10.4000/assr.3790 


\section{Youssef Belal}

\section{Mystique et politique chez Abdessalam Yassine et ses adeptes}

Un lieu commun de la science politique veut que ce que l'on appelle « islamisme », s'oppose au soufisme ${ }^{1}$. Pourtant, le mouvement " Justice et bienfaisance » (al-'adl wa al-ihsân) qui est régulièrement l'objet de l'attention médiatique au Maroc semble contredire ce postulat. Je souhaiterais ici m'écarter de la manière dont la science politique traite habituellement le phénomène dit "islamiste » (elle s'intéresse avant tout à la question de la prise du pouvoir) et plutôt me pencher sur quelques aspects des fonctions d'un mouvement présenté comme " islamiste ", c'est-à-dire sur l'attrait, l'intérêt, la satisfaction ${ }^{2}$ que peuvent avoir des femmes et des hommes à s'identifier à ce mouvement et à prendre part à son existence collective ${ }^{3}$. Les tensions entre les différentes représentations de l'islam et du monde que se font les hommes et les femmes qui appartiennent à ce mouvement sont en fait révélatrices des tentatives de conciliation entre des formes, qu'on a souvent présentées comme opposées, de la religion et de l'existence. Ces tensions sont perceptibles à plusieurs niveaux : entre la tentative de compromis avec le monde et son rejet, entre la Loi et la mystique, entre l'individu et le collectif, entre la médiation des hommes (incarnée en un seul homme) et celles des textes (ceux du Coran et de la Sunna), et enfin entre l'émotion religieuse (ou extatique) et les attentes de bénéfices mondains (ou sacré social).

1. Je dois ici remercier Houari Touati et Isabelle Rivoal qui ont lu ce texte et m'ont fait part de leurs commentaires.

2. Je souhaite ici m'intéresser tout particulièrement à la Weltanschauung (au sens de Dilthey) qui anime ce mouvement. Cf. Wilhelm DiLTHEY, Conception du monde et analyse de l'homme depuis la Renaissance et la Réforme, Paris, Cerf, 1999.

3. Je pense ici à la démarche de Max Weber, "L'expérience religieuse en tant que telle est évidemment irrationnelle comme toute expérience ; [...] mais cette irrationalité n'empêche pas qu'il soit de la plus haute importance, en pratique, de savoir quel type de système de pensée confisque pour ainsi dire le vécu religieux immédiat et lui impose ses schémas ". Max Weber, L'Éthique protestante et l'Esprit du capitalisme, Paris, Flammarion, 2000, p. 16. 
Une analyse ethnographique ${ }^{4}$ du mouvement, en parallèle à une lecture ethnographique des textes s'inspirant de la méthode herméneutique ${ }^{5}$, peut nous montrer comment un projet politique de transformation du réel peut être porté par un mouvement qui, dans son fonctionnement quotidien, est avant tout un mouvement mystique qui s'accommode du monde tel qu'il est. La dimension révolutionnaire du projet politique est elle-même significative de la tension qui traverse le mouvement entre compromis avec le monde et rejet du monde. Le projet politique initial d'A. Yassine montre bien que le mouvement entend être dans le monde mais à la condition de le changer. À se limiter au texte ou au rapport du mouvement au champ politique, on serait tenté de croire que les adeptes d'A. Yassine ne cherchent que le changement politique. À se limiter à l'étude du fonctionnement quotidien et des rituels, on pourrait croire que c'est uniquement un mouvement mystique. Si on intègre la dimension temporelle, cette tension, au fil du temps, est régulée au profit d'un compromis avec le monde car confronté au réel, le projet politique tend à s'essouffler au profit de la dimension spécifiquement religieuse de médiation. En tout cas le projet politique (dans le sens d'un projet dirigé contre l'État et qui vise le pouvoir) n'est pas structurant dans le fonctionnement quotidien du mouvement et c'est une explication bien faible de ce qui pousse les femmes et des hommes à y adhérer et à être assidus aux rituels collectifs.

Au niveau de la pratique spécifiquement religieuse, les adhérents vont trouver en A. Yassine un entrepreneur religieux qui tente de construire sa pratique mystique sur ce qu'il estime être les normes cultuelles et sociales prescriptives (dans une certaine mesure "légales ») de l'islam. Ces individus qui se veulent membres de la Jamâ'a appartiennent généralement à la classe moyenne instruite (professions dans l'enseignement, l'administration, petits entrepreneurs, quelques professions libérales, étudiants...) soit par leur statut hérité, soit par ascension sociale, soit par déclassement social, et ce sont tous des profanes (ou des "laïcs »). Pour nombre d'individus qui sont donc partie prenante de processus économiques (travail) et sociaux (famille, études), la vie mondaine est loin de coïncider avec la vie extra-mondaine du groupe, et l'arbitrage temporel qu'ils peuvent faire entre les deux est toujours incertain. Par ailleurs, par leur

4. Il me paraît indispensable d'effectuer ce que Geertz appelle à la suite de Gilbert Ryle, la «Thick description». "Doing ethnography is like trying to read a manuscript [...]. It is interpretive; what is interpretive is the flow of social discourse, and the interpreting involved consists in trying to rescue the said of such discourse from its perishing and fix it in perusable terms [...]. There is, in addition a fourth characteristic of such description, it is microscopic ». " Thick Description: toward an Interpretive Theory of Culture », in Clifford GEERTZ, The Interpretation of Cultures, Londres, Fontana Press, 1993, p. 10.

5. Alton Becker, à propos du théâtre d'ombre indonésien, écrit : " 1) The relation of words, phrases, sentences and larger units of the text to each other (the coherence of the text). 2) The relation of this text to other texts, the extent that it is repetition or new (speaking the present or the past). 3) The relation of the author to both the text and the hearers/readers of the textseen from the point of view of the author or from the point of view of the hearers/readers (the intent of the text-builder). 4) The relation of units in the text to non-literary events (reference) ", Alton BeCKer, Yengoan, eds., Text-Building, Epistemology and Aesthetics in Javanese Shadow Theatre, in The Imagination of Reality, Norwood (NJ), Ablex, 1979, p. 159. 
éducation, ils ont suffisamment de bases pour avoir une pratique cultuelle autonome et ils sont théoriquement en mesure d'avoir un accès direct aux textes fondateurs en y consacrant le temps suffisant. Pourtant, s'identifier à la Jamâ'a et adhérer à une pratique collective de la religion c'est reconnaître à A. Yassine le statut de médiateur entre Dieu et les hommes. Enfin la pratique des rites ( $d h i k r$, nasîha, ziyâra, i'tikâf) à plusieurs échelles (quartier, district, région, province, territoire national et domicile d'A. Yassine qui est aussi le centre exemplaire) sont des moments qui permettent aux membres du mouvement de ressentir une émotion religieuse qui varie en intensité. Ces instants parfois quasi-extatiques où le croyant entre en relation avec le monde du " mystère » (al-ghâyib), répondent à des besoins qui doivent être conciliés avec l'attente de bénéfices matériels dont peut être pourvoyeur A. Yassine grâce à sa baraka.

\section{La ziyâra (visite) à Salé}

A. Yassine se fait connaître, dès 1974, en envoyant une épître à Hassan II lui enjoignant de rejoindre " la voie droite ». Il fait de cet acte son entrée sur la scène politique après avoir quitté une confrérie soufie, la tarîqa budshishiyya à laquelle il avait adhéré au moment où sa vie d'instituteur, puis de directeur du centre de formation des inspecteurs du ministère de l'Éducation nationale, ne le satisfaisait plus. Le crime de lèse-majesté lui vaut de la part du monarque quelques années en asile psychiatrique. Fort de ce capital symbolique, il s'attelle dans les années 1980 à organiser le mouvement qu'il crée en 1981. Il alterne périodes d'emprisonnement et de résidence surveillée qui contribuent à faire de lui un homme de courage et de persévérance, qualités indispensables à un homme de baraka. Ces moments d'isolement sont aussi pour lui l'occasion d'écrire de nombreux ouvrages dans lesquels il se pose en rénovateur de l'islam et où il expose ce que doit être la "voie prophétique ", al-minhâj an-nabawî.

À partir du milieu des années 1980, la Jamâ'a commence à croître de manière conséquente et devient incontournable dans les universités après les affrontements avec les étudiants gauchistes à Fès et à Oujda à la fin de 1991. D'autres événements sont l'occasion, pour la Jamâ'a, de montrer sa présence dans l'espace public: guerre du Golfe en 1991, grèves étudiantes, projet de réforme du statut de la femme par le gouvernement socialiste de Abderrahman Youssoufi, conflit israélo-palestinien... Quelque temps après la mort de Hassan II, A. Yassine ne manque pas d'envoyer une nouvelle épître au nouveau roi en 2000 dans laquelle il l'appelle à restituer la fortune de son père au peuple marocain ${ }^{6}$. En mettant fin

6. Pour une présentation du mouvement, il convient de faire référence à Mohammed TozY, Monarchie et Islam politique, Paris, Presses de Sciences-po, 1999 et à Mohammed DARIF, Jamâ'at al-'adl wa al-ihsân, qirâ'a fi al-masârât, Casablanca, Éditions de la Revue marocaine de science politique, 1992. Pour une analyse de la pensée d'A. Yassine on peut lire Mohammed Cherkoun, "Islamisme, messianisme et utopie au Maghreb ", Archives des sciences sociales des religions, $\mathrm{n}^{\circ}$ 75, 1991 et aussi Mohammed EL AYADI, «Abdessalam Yassine ou le poids des paradigmes ", in Aïssa KADRI, Parcours d'intellectuels maghrébins, Paris, Karthala, 1999, p. 129-163. 
à la résidence surveillée la même année, la monarchie va permettre à Abdessalam Yassine de faire une tournée dans tout le Maroc et de recevoir les visites de ses adeptes à Salé.

La maison d'A. Yassine, située à Salé, est comme une insolence à la face de la monarchie dont le Palais et le mausolée se trouvent à Rabat, sur l'autre rive du Bu Regreg qui sépare la capitale de l'ancienne ville pirate ${ }^{7}$. Sa demeure constitue sans nul doute pour les adeptes du mouvement un pôle de sainteté, lieu pourvoyeur de baraka. Elle est devenue un lieu sacré, une sorte de horm dont l'accession est soumise à un rituel rigoureux. Toute personne qui désire rendre visite à "Si Abdessalam » doit en faire la demande deux ou trois semaines à l'avance ${ }^{8}$. Une fois la demande formulée et acceptée, un ticket numéroté qui sera demandé le jour de la ziyâra est donné à la personne avec la date de la visite. Le terme de ziyâra est utilisé dans le langage courant pour désigner une simple visite que l'on rend à des membres de la famille ou des proches, mais il est aussi utilisé pour les visites de tombeaux de saints ou pour des pèlerinages locaux. Il peut parfois désigner le don destiné au saint. Parler de ziyâra connote donc le caractère proche d'A. Yassine et son rôle de walî, ami et protecteur. Cette ziyâra, qui est aussi une séance de $d a^{c} w a \hat{a}$ (prédication) et de nasîha (conseil), est généralement le moment où $\mathrm{A}$. Yassine ou ses compagnons fondateurs du mouvement vont prêcher la bonne parole devant un auditoire composé d'adhérents ou de sympathisants du mouvement venus des grandes villes du Maroc avec une majorité de jeunes (moins de trente-cinq ans). La direction du mouvement fait d'ailleurs en sorte que les personnes présentes soient issues de l'ensemble du Maroc et désormais du "monde » en diffusant le film de la séance sur internet et en recueillant les questions venues d'Amérique ou d'Europe. La séance se déroule généralement le dimanche à partir de onze heures du matin.

Le lieu où se déroule la séance n'est pas en réalité la demeure de A. Yassine. Dans un quartier de la nouvelle ville de Salé, trois villas dont l'une est le gîte du murshid (guide) sont concentrées dans la même rue et sont utilisées par le mouvement pour y accueillir un seul sexe ou pour un usage mixte (ce qui est le cas de la ziyâra). Femmes et hommes entrent par la même porte et présentent le ticket donnant le droit de visite. Une fois à l'intérieur, les hommes sont dirigés à droite de la porte principale tandis que les femmes sont guidées vers la gauche. Venus du monde de l'impur, entrant dans un lieu rituellement purifié, hommes et femmes doivent, comme s'ils entraient dans une mosquée ou dans le tombeau d'un saint, déposer leurs affaires à l'entrée, se déchausser et faire leurs ablutions. Dans une atmosphère de silence et de recueillement, les hommes, une fois entrés dans le monde de la pureté, se dirigent vers une pièce sans meubles aménagée

7. Sur l'histoire de Salé on peut se reporter au livre de Kenneth Brown, People of Salé, Cambridge (Mass.), Harvard University Press, 1976.

8. J'ai pris part à cette ziyâra en été 2003. Je remercie tout particulièrement Abu al 'Alâ' N. sans qui ce terrain n'aurait pas été possible. 
pour recevoir le plus grand nombre de personnes. L'angle de la pièce recouvert d'un rideau bleu disposé juste derrière le sofa sur lequel vont s'asseoir les hommes pieux produit un contraste saisissant avec les murs peints en blanc entourant un sol sans meubles où les visiteurs vont s'asseoir sur des tapis confortables disposés à même le sol. L'angle de la pièce marque aussi la limite entre les hommes assis à la face gauche du sofa et les femmes à sa face droite, tous disposés en rangées de six à sept personnes. Cette topographie de la ziyâra permet de voir que celui qui va prêcher la bonne parole est à la fois la frontière et le lien entre les hommes et les femmes. Il peut parler aux deux sexes, il peut comprendre les femmes et les hommes. Ceux qui lui rendent visite sont de facto des gens d'humilité, des disciples qui, assis en tailleur à même le sol, vont écouter la nasîha de celui qui va leur parler du haut du sofa. Pour cela femmes et hommes doivent attendre leur protecteur et se concentrer. Ils doivent être présents à onze heures pour le voir et l'écouter à partir de onze heures trente. Ils doivent fortifier leur imân (foi), en attendant la venue de "Si Abdessalam ", en pratiquant le dhikr et en lisant le Coran silencieusement. C'est d'une certaine manière s'apprêter à être mis en relation avec l'autre monde grâce au médiateur qui est aussi un guide pour la vie d'ici-bas.

Finalement cette fois ce ne sera pas Si Abdessalam, souffrant, qui parlera, mais ses compagnons de la première heure, ceux qui ont créé le mouvement avec lui lorsqu'ils n'étaient encore que trois. Son absence n'est que physique et tout le monde pense à lui. Les deux compagnons fidèles sont d'ailleurs là pour dire ce qu'il dit, pour parler de lui et de la Jamâ'a.

\section{Écoutons un instant le premier compagnon d'A. Yassine}

Les actes ne valent que par les intentions (innama al-a'mâl bi al-niyyât). Il est impératif de conserver la bonne intention, d'effectuer la visite avec la bonne intention et nous souhaitons la guérison à Si Abdessalam. Souhaitons longue vie au guide et implorons Dieu de protéger la Jamâ'a du mal. Il faut voir l'avenir avec miséricorde (rahma). La Jamâ' $a$ est une école pour apprendre notre religion et nous resterons des élèves fidèles du guide en étant repentis, engagés, sincères, et en ayant la volonté ( raghba). La chose la plus haute c'est voir le visage de Dieu le jour dernier. Dans cette école vous sentez la sérénité (tuma'nîna), et la fortune (tawfîa) de notre maître alors que les gens vivent dans la perdition (ghafla). Souriez à la vie et c'est à vous de faire votre travail intérieur. Comme le dit Si Abdessalam le malheur a vaincu les gens. Notre voie est évidente. La voie prophétique s'offre à nous. Nous avons choisi la voie du hadîth, et de la Sunna. Nous allons sur la voie, il ne faut pas ressentir l'obscurité. Notre voie est claire (tarîqunâ wâzih). La voie du Prophète est évidente. S'ils se sont dirigés vers les livres, les revues et les télévisions, nous avons préféré le Livre de Dieu et la Sunna du Prophète. Ainsi vécurent ses compagnons. Comme le dit le guide, la route et la voie sont claires. Nous voulons dans cette Jamâ'a restituer l'évidence de cette voie (wuzûh at-tarîq) par le compagnonnage (subba) et dans les séances (majâlis). Le savoir comme le dit le docteur Qaradhaoui ${ }^{9}$ ce ne sont pas les idées ou l'information, c'est la lumière,

9. Youssouf al-Qaradhaoui est l'ouléma vedette de la chaîne Al-Jazira, régulièrement invité de l'émission « la sharî̀ $a$ et la vie ». 


\section{i70 - ARCHIVES DE SCIENCES SOCIALES DES RELIGIONS}

(an-nûr) en imitant le Prophète, c'est cela l'essence du savoir ( $r u b$ al-'ilm). Il faut dire "Dieu donne-moi le savoir ", il faut demander la perfection (kamâl), la foi et le savoir. L'amour du Prophète nous amène à s'imprégner de ses mots et de ses comportements. Il faut éviter les comportements individuels autant que possible. Parfois on peut être tenté de les adopter. La Jamâ'a et le compagnonnage permettent d'avoir le lien avec Dieu et avec ses adorateurs. C'est notre lien avec Dieu (râbita 'ubudiyya). C'est une relation double mais aussi une relation avec les parents, les orphelins, l'épouse, les voisins. La terre de l'impiété $(k u f r)$ est en fait une terre d'appel (da'wa), car c'est la condition de l'homme que de faire l'appel à Dieu. Il faut éviter les jugements catégoriques, l'excommunication, l'humiliation des gens et de montrer du doigt les défauts. Le Prophète était miséricordieux (yarhamu). Si nous avons une incompréhension il faut la résoudre par le spirituel (ruhî) et par le cœur. Dans les leçons, vous avez appris que le savoir véritable est un savoir du cœur (qalbî) et que c'est par le compagnonnage que l'on peut atteindre les lumières de la voie (anwâr al-huda) et la foi. C'est pourquoi le compagnonnage est impératif et les séances d'éducation (majâlis tarbawiya) et de conseil (nasîha) servent à cela. L'éducation est au-dessus des attaques du pouvoir. Face à la dureté de la discorde, ceux qui se rangent derrière Dieu sont les plus forts. Le compagnonnage est une question de vie ou de mort pour nos cœurs mêmes. S'éloigner des croyants cela signifie le désespoir. Le compagnonnage des hommes de piété et de bien (as-sâlihîn) est une condition pour sortir la discorde des cœurs. Personne ne peut se passer du compagnonnage et de la Jamấa. Le compagnonnage c'est suivre la Sunna et le Livre. Il ne faut pas oublier la piété filiale (al-bar bi al-wâlidayn). La plus grande des fautes consiste à la commettre contre sa mère.

Le moment des questions venu, un jeune homme de Casablanca prend la parole: "Je n'ai plus de temps pour la $d a^{\prime} w a$ car je dois me consacrer à mes études. J'ai mauvaise conscience car je sais que c'est impératif. Je vous demande une prière pour ma mère ». Un adhérent de la $\operatorname{Jamâ}^{\prime} a$, la quarantaine, venu de Oujda, ville de l'est du Maroc, fait part de sa mauvaise conscience : "Je suis inquiet car j'ai moins de temps pour assister aux majâlis". Une jeune femme d'une trentaine d'années pose la question suivante : "Comment peut-on arriver à l'ihsân ?".

Le compagnon de route d'A. Yassine répond : "La da'wa est une responsabilité grandiose pour les femmes et les hommes. Après plus de vingt ans de prison et de blocus, malgré ces obstacles, la Jamâ'a a surmonté les difficultés. C'est une Jamâ'a qui a la protection de Dieu ". À ce moment le disciple des premiers temps, aujourd'hui âgé de plus de soixante-dix ans, pleure. Il reprend: "Comment entretenir cette flamme ? La da'wa est une traduction de ce qu'il y a dans le cœur. Face à la jeunesse perdue la da'wa permet de constituer une coupure avec le mal et le divertissement. On a appris du guide que le croyant est celui qui combat son ego $(n a f s)$ car l'ego c'est le mal... Dieu aime le repentir et les repentis. La séance de conseil, majlis an-nasîha est un grand bien, irremplaçable car il permet de se repentir... Il y avait autrefois un jeune qui était étudiant très actif. Après la licence il devint instituteur, et il se fait de plus en plus rare. Lorsque le guide me demanda ce qu'il est devenu je lui ai dit que je n'avais pas de nouvelles depuis cinq années. Il a été envahi par d'autres habitudes. Il s'est trouvé loin de Dieu. L'éloignement est une question d'habitudes. Lorsqu'il a commencé à 
gagner de l'argent, à avoir une maison et une voiture il est entré dans un monde à l'opposé du monde de la foi. C'est une faute grave (ma'siya), car c'est s'éloigner de la vérité de la foi et préférer le commerce et le divertissement au compagnonnage.

Une semence peut donner naissance à un arbre. Avec les litanies $(d h i k r)$, le compagnonnage et les séances on peut comprendre ce qu'est l'homme irrigué par la lumière (nurâni). Nous ne voulons pas les élections ou le Parlement car nous suivons la voie des hommes bienfaisants (sabîl al-mubsinîn). Il y a plus de trente ans M.S. voulait une mort facile au moment du jihâd en Afghanistan. M. S. est venu trouver Si Yassine pour lui dire qu'il allait partir pour une mort facile. Si Yassine lui a dit que "la plus haute des morts est celle dans la da'wa avec les frères et les sœurs ».

Après la seconde intervention du compagnon de route d'A. Yassine, une femme demande des prières $\left(d u^{\prime} \hat{a}^{\prime}\right)$ pour sa guérison. On s'enquiert de l'état de santé du guide. Puis c'est au tour de ceux qui ont une vision ou un rêve (ru'yia) à raconter, de parler. Un homme de Safi, pêcheur et père de trois filles raconte : "Après les événements du 16 mai, les croyants étaient réunis pour un majlis (séance). Le walî vint à leur rencontre et c'était en fait pour venir participer au majlis. La terre entière était devenue mosquée ». Une femme raconte le rêve qu'elle a eu : "Après les événements du 16 mai $^{10}$, j'ai eu la vision qu'on allait avoir la visite de Si Abdessalam. Mais on avait peur qu'on vienne nous emprisonner. Avec Si Abdessalam ils ne nous ont pas approchés ».

Le compagnon de route d'A. Yassine met fin à la ziyâra en faisant des prières $\left(d u^{\prime} \hat{a}^{\prime}\right)$ : " pour la mère de ce jeune homme, pour cette femme souffrante, pour résoudre les problèmes de cette autre femme, pour le rétablissement de $\mathrm{Si}$ Abdessalam, pour cette Jamấ $a$, pour l'ensemble des musulmans ».

\section{A. Yassine walî, médiateur entre Dieu et les hommes}

A. Yassine est au centre de la Jamấ $a$, celui vers lequel tous convergent, celui par qui la relation avec Dieu est possible. Entretenir sa foi en pratiquant les cinq piliers est une chose nécessaire. Mais elle ne peut suffire à elle seule et la subba, compagnonnage, sur laquelle repose la Jamấa et la relation à Si Abdessalam va permettre de trouver la bonne voie. Seule cette subba va permettre de se libérer du malheur et de cette nuit noire qui obscurcit la vie des hommes et des femmes. Grâce au murshid et à la Jamấa, la voie est évidente. Partant de là, il faut être confiant dans l'avenir car si la voie est évidente il faut juste un guide qui, sur le modèle du Prophète, va enseigner le vrai savoir. Ce 'ilm ce n'est pas l'érudition ou le savoir désincarné des livres mais la lumière, nûr, dont l'homme sera irrigué en imitant le Prophète. Imiter le Prophète c'est en définitive imiter Si Abdessalam

10. Le 16 mai 2003 des kamikazes qualifiés de "salafistes-jihadistes " par la presse ont commis, à Casablanca, un attentat à la bombe qui a causé la mort de quarante-cinq personnes. 
qui sait restituer aujourd'hui aux hommes qui veulent le suivre dans la Jamâ'a le modèle du Prophète, c'est-à-dire celui qui rend possible l'acte d'être ${ }^{11} \mathrm{du}$ Prophète. Aimer le Prophète et s'imprégner de sa lumière et de son être c'est donc hic et nunc aimer A. Yassine qui rend possible la relation des hommes avec le Prophète modèle des hommes et figure des hommes la plus haute chez Dieu. La Jamâ'a est en définitive une râbita 'ubudiyya, le lieu de l'adoration de Dieu puisqu'elle est, grâce à A. Yassine, la médiatrice entre Dieu et les hommes.

Sa qualité de saint ${ }^{12}$ ne fait aucun doute. Elle est prouvée par sa capacité à survivre aux plus grandes épreuves, par son courage exemplaire et par sa persévérance. A. Yassine est celui qui a envoyé trente ans plus tôt une épître à Hassan II où il l'enjoint de rejoindre la voie droite, juste après les tentatives ratées de putsch contre la monarchie c'est-à-dire à un des moments les plus noirs de l'histoire du Maroc. Les derniers moments de la séance qui sont, comme nous l'avons vu, réservés à un très bref récit des rêves, $r u^{\prime} y a$, illustrent bien comment les adhérents de la Jamâ' $a$ attendent d'A. Yassine une protection face à la prédation de l'État patrimonial, le makzhen. Le premier récit permet aussi de traduire les tensions vécues par certains adhérents dans leur rapport au monde. Rappelons que le wali, qui est une sorte de gouverneur de région, est le représentant du makhzen au niveau local. Il est d'ailleurs intéressant de relever que le terme peut désigner aussi quelqu'un comme A. Yassine qui est wali Allah, un ami de Dieu. Si le walî vient au majlis, cela signifie que le mouvement est devenu allié (ici aussi on peut utiliser le terme walî) du makhzen et qu'il n'est plus en situation de semi-clandestinité. Dans ce cas de figure il n'y a plus de tension entre la voie de la Jamâ'a et le monde tel qu'il est ici symbolisé par le makhzen. Cela aussi peut vouloir dire que certains adhérents vivent mal la légitimité concurrente d'A. Yassine face à la monarchie et préfèrent une coexistence heureuse à une confrontation larvée. Mais le fait que la terre entière était devenue mosquée ${ }^{13}$ peut également signifier que le mouvement a réalisé sa vocation de da'wa et que la monarchie elle-même représentée par le walî se soumet à l'autorité d'A. Yassine. Ce récit montre bien la tension entre la situation de semi-clandestinité du mouvement surtout après les attentats du 16 mai et la vocation du mouvement à s'étendre par sa mission de da'wa.

Cette tension contribue à faire de l'allégeance à A. Yassine un acte vital pour les adeptes. Pour suivre sa voie et son enseignement, il est indispensable de faire preuve de sidq, c'est-à-dire que l'adepte doit suivre tout ce que lui prescrivent la Jamâ'a et son guide. Ceux qui veulent suivre la voie du Prophète, c'est-à-dire

11. Je reprends ici un concept utilisé par Christian JАмвет, L'acte d'être. La philosophie de la révélation chez Molla Sadra, Paris, Fayard, 2002.

12. Pour une étude d'ensemble de la sainteté au Maghreb, on peut se reporter à Émile Dermenghem, Le culte des saints au Maghreb, Paris, Gallimard, 1982.

13. La terre comme mosquée est un hadith du Prophète souvent cité par les musulmans. 
la voie d'A. Yassine, doivent être conscients que l'ego, le nafs, peut être un obstacle à tout moment. Il faut combattre le nafs qui est le mal (su'). Valoriser son nafs est en fait incompatible avec le rôle assigné à la Jamấa et à A. Yassine. Il faut être capable de se dévouer pour la Jamâ $\hat{a}^{\prime} a$ et pour être réceptif à l'enseignement du maître il faut que l'âme soit vierge de l'ego. Laisser le nafs triompher c'est avoir le destin de cet instituteur qui a pris goût à la vie matérielle et qui a préféré le divertissement $(l a h w)$ à la subba en se laissant envahir par d'autres habitudes. Il faut au contraire demander d'achever sa vie parmi « les frères et les sœurs» car la mort dans la da'wa est bien plus haute que celle dans le combat armé.

Souhaiter cette mort parmi cette famille qu'est la Jamấa révèle son caractère sacré. Personne collective, elle doit tout à A. Yassine. Elle constitue la forteresse contre le chaos de la ville moderne. Elle protège des maux que sont l'individualisme, l'anonymat, la violence des rapports sociaux et l'hostilité de l'autre. En un mot les hommes vivent dans la perdition, la ghafla. Le modèle du lien social n'est-il pas celui de la famille idéale qui est le lieu de l'amour et qui protège les hommes des infortunes de la vie ? C'est pourquoi le plus grand des devoirs est la gratitude envers les géniteurs et tout particulièrement la mère. Mais rappeler aux hommes le devoir de gratitude envers le père et la mère c'est demander de les protéger et c'est donc reconnaître qu'à eux seuls ils ne peuvent être des protecteurs efficaces. Avoir avec Si Abdessalam et avec la Jamấa le même rapport de respect, d'abnégation et d'amour est chose normale puisque leur protection est, contrairement à celle de la famille biologique, efficace. La Jamấa est un lien entre les hommes, elle incarne le lien de l'islam face à la dislocation du lien social. Un homme sans la Jamâ'a, sans le compagnonnage et sans guide, est un homme perdu, victime de la fitna. Face à la jeunesse perdue, la «da'wa, appel, permet de constituer une coupure (qatîे $a$ ), avec le divertissement et le mal ». Rendre effective cette mission de da'wa c'est ériger le compagnonnage et l'acte de plaire à Dieu (tahabbub) en modèle de relation avec la micro-société dans laquelle les hommes vivent. C'est pourquoi il faut rester « des élèves fidèles du guide, (talâmid awfiya') » d'autant plus que A. Yassine "apporte sérénité et bonne fortune (tuma'nina et tawfîq)».

Le conseil (nasîha) du compagnon d'A. Yassine n'est donc valide que parce qu'il est son élève fidèle et son délégué. Il vient dire ce que A. Yassine dit. Il fait de manière récurrente référence à lui. Cette délégation de la nasîha à des représentants d'A. Yassine est institutionnalisée par une organisation du mouvement à l'échelle du quartier (la cellule familiale, usra), du district (shu'ba), de la province (iqlim) et de la région (jiha). Ces représentants sont aussi en quelque sorte les garants de la transmission de la baraka d'A. Yassine. Par ce type d'organisation, le mouvement épouse la géographie urbaine du Maroc et confère à A. Yassine une certaine ubiquité. 


\section{Bonne conscience et action mondaine}

Cette relation de l'adepte avec le guide et la Jamâ'a que l'on vient de décrire reste en bonne partie une relation idéale. En fait, les individus, forts de leur sentiment d'appartenance à la Jamâ'a, vont aussi lui déléguer leur pratique religieuse, notamment surérogatoire, afin de mieux se consacrer aux affaires du monde. En ce sens, la niyya est une notion centrale pour comprendre ce paradoxe.

Faire de la niyya le critère de jugement de toute action ( «les actions ne valent que par les intentions » est un dire du Prophète) c'est accorder toute son importance à la vie intérieure ${ }^{14}$ et refuser d'accorder la primauté à l'aspect extérieur des choses. Les actes ont toute leur importance à condition que la niyya, la bonne intention, en soit à l'origine. Or cette bonne intention est elle-même le fruit d'un travail sur soi qui va permettre d'être repentant, pieux et volontaire et qui doit permettre de tendre vers l'ihsân, bel agir. L'exemple de la jeune femme qui demande la voie à suivre pour arriver à l'ihsân montre bien comment les questionnements mystiques sont très présents dans les préoccupations des adeptes. Chez les soufis, l'ihsân, qui demande de se libérer de soi-même pour tenter de voir le visage de Dieu, est le degré le plus haut de réalisation de soi. C'est l'étape la plus haute après l'islam qui consiste à pratiquer les cinq piliers et l'imân qui est la foi en Dieu. Chez A. Yassine toute action doit tendre vers cet idéal d'ihsân et c'est à dessein que le mouvement se nomme al-'adl wa al-ihsân. Le fait que cette jeune femme pose cette question lors de la ziyâra illustre bien que même les préoccupations spirituelles et mystiques, celles qui, $a$ priori relèveraient de l'intériorité, doivent avoir une réponse concrète, apportée par un homme qui est le lien avec l'autre monde.

Toutefois il faut bien voir que la niyya a un autre versant. Couplée à la notion de savoir, 'ilm, comme lumière et comme langage du cœur, elle permet à des individus qui sont dans la vie mondaine et qui consacrent l'essentiel de leur temps à des affaires profanes (travail, études, famille...) d'avoir bonne conscience aussi bien dans leur rapport à la Jamâ' $a$ et au temps qu'ils lui consacrent que dans leurs affaires spécifiquement mondaines. La mauvaise conscience que certains membres expriment est très révélatrice de la fonction que remplit pour eux aujourd'hui la Jamấa $a$. Lors de cette ziyâra, la préoccupation majeure des femmes et des hommes présents tournait en effet autour du temps consacré à la pratique religieuse la plus haute à leurs yeux qui est la pratique religieuse collective dans le cadre de la Jamấ $a$. Un rapport individuel à Dieu, sans médiateur, et cantonné aux cinq piliers et à la Loi est trop abstrait, trop soumis au doute et à l'incertitude pour tous ceux qui sont dans la vie mondaine. Or, la vie urbaine est une vie

14. Comme le rappelle Claude Levi-STrauss, " la preuve du social, elle, ne peut être que mentale; autrement dit nous ne pouvons jamais être sûrs d'avoir atteint le sens et la fonction d'une institution si nous ne sommes pas en mesure de revivre son incidence sur une conscience individuelle. " in Marcel Mauss, Sociologie et Anthropologie, Paris, PUF, 1966, p. XXVI. 
matérielle qui est particulièrement chronophage et beaucoup d'adeptes ont le sentiment qu'ils ne consacrent pas suffisamment de temps à la Jamâ'a. Le cas de ce jeune étudiant devenu instituteur qui a tourné le dos à la Jamâ'a et qui a fini par se consacrer pleinement à la vie mondaine est précisément l'exemple à ne pas suivre. Le problème est de concilier son identité d'individu intégré dans des processus économiques et sociaux avec l'identification à un corps collectif qu'est la Jamâa $a$. Cette tension se traduit par une tentative de conciliation entre d'une part, la pratique religieuse individuelle et individualisée, plus conforme à un cadre moderne et urbain qui va souvent de pair avec un islam universel, scripturaire et démocratisé dont on attend généralement peu de bénéfices matériels et d'autre part, une pratique religieuse collective qui se fait dans la Jamấa grâce à un médiateur entre Dieu et les hommes, capable de traduire la grâce divine en ces bénéfices. Au-delà de la médiation, il y a même une délégation de la pratique religieuse. En valorisant son caractère collectif, la Jamâ' $a$ a aussi pour effet paradoxal de contribuer à la circonscrire aux rites collectifs : $d h i k r$, lecture collective du Coran, i'tikâf... Cette délégation à un corps collectif et la croyance en la niyya permettent ainsi de mieux se consacrer aux affaires du monde d'autant plus que la baraka d'A. Yassine permet d'espérer des bénéfices matériels de cette médiation. En ce sens, les attentes des femmes et hommes présents au moment de la ziyâra peuvent parfois être aussi explicitement concrètes. La séance de nasîha se termine par exemple avec l'accomplissement de $d u^{\text {' }} \hat{a}$ ' demandée par une femme pour résoudre "ses problèmes » tandis qu'une autre femme souffrante demande une prière pour sa guérison.

\section{«La voie prophétique "}

Nous avons vu dans la première partie de notre analyse que la médiation religieuse dans le mouvement d'A. Yassine se révèle être, pour une bonne part, une délégation de la pratique religieuse. Dans cette seconde partie, je souhaiterais montrer que cette médiation religieuse est indissociable chez A. Yassine d'une médiation politique. Pour bien comprendre ce qu'il attend de cette allégeance politique, il convient de lire quelques passages de son livre majeur al-minhâj an-nabawî qui est enseigné aux adeptes en tant que discipline religieuse ${ }^{15}$ au même titre que des versets du Coran, des hadith-s ou du fiqh.

Cette lecture a, par ailleurs, d'autres avantages. En se penchant sur les références qu'il utilise on peut savoir quelles sont ses sources d'inspiration et le situer dans l'histoire intellectuelle à laquelle il se rattache. On peut surtout comprendre comment A. Yassine va charger les termes anciens qu'il utilise d'un sens nouveau et quelles sont les ruptures qu'il apporte par rapport aux références qu'il utilise.

15. La matière enseignée dans les majâlis s'intitule al-fikr al-minhâjî̀, la pensée «minhajienne ". 
En second lieu, on peut mesurer l'écart entre un projet initial cohérent parce que couché sur le papier (la première édition de son livre al-minhâaj an-nabawî date de 1981-1982) et la réalité traversée par des tensions voire des contradictions. Cette lecture de la réalité à la lumière du texte et inversement du texte à la lumière de la réalité permet de rendre intelligible ce qui ne l'est pas toujours au premier abord. Cette démarche est par ailleurs indispensable pour comprendre les ruptures de sens situées dans le contexte où elles font sens ${ }^{16}$ car, comme l'a montré Jack Goody, la « raison graphique » permet précisément de décontextualiser le texte des circonstances de sa production ${ }^{17}$.

Le titre est en lui-même significatif. Il apparaît comme en écho à la parole de Dieu, Likulin ja'alnâ minkum shir'atan wa minhâja, "À chacun de Vous, Nous Vous avons fait parvenir des prescriptions et une voie " ${ }^{18}$, comme si A. Yassine n'en avait isolé que la seconde partie. En définitive, la sharî‘ $a$ devient seconde, voire se subsume dans le minhâji, à la manière de Qotb, le penseur phare des Frères musulmans, qui parlait de fiqh harak $\hat{\imath}^{19}$ dans son commentaire du Coran.

Al-minhâj al-nabawî se compose de deux grandes parties. Une première partie (les quatre premiers chapitres) pose à la fois la finalité du mouvement (Revivifier la religion et la foi, réaliser le soulèvement et créer l'État islamique qui ne sont que se rapprocher de Dieu) et les principes sur lesquels repose toute action du mouvement (l'éducation, tarbiyya et l'organisation, tanzîm). Dans la seconde partie, A. Yassine entend montrer la manière de réaliser les objectifs et d'être fidèle aux principes exposés dans la première partie. Pour cela il présente dix sections qui sont les dix qualifications pour revivifier la foi (al-khisâl al 'ashar), qui commencent par la subba et la jamâ'a pour culminer dans le jihâd comme principe de vie.

Comment situer le livre d'A. Yassine ? Essayons d'abord de voir ce qu'il n'est pas. Il ne prend pas la forme d'un recueil de fatwas, ni d'un livre de fiqh, ni d'un livre sur les sources du droit, usul al-fiqh, et ce n'est pas un commentaire,

16. Jack Goody, La raison graphique, Paris, Éditions de Minuit, 1986.

17. L'étude de Brinkley Messick dans le cas du Yémen est une des rares tentatives de saisir les ruptures de sens et de comportement introduites par l'imprimerie et l'éducation moderne dans le rapport au savoir religieux et dans la transformation des rapports de pouvoir. "While recitation was thought to maintain a reliable constancy of meaning, the secondary medium of writing was seen as harboring a prospect of misinterpretation. [...] Extending the authoritygiving presence of an original author to other places and times would be the work not only of recitational devices but also of writing. In written form, however, the general misreadability of the medium was dangerously extended by the open potentiality of the texts themselves ". Les lectures décontextualisées sont en quelque sorte la vocalisation de textes sans voyelles. Cf. Brinkley Messick, The Calligraphic State, Berkeley, University of California Press, 1993, p. 25-26.

18. Sourate $A l-M a ̂$ 'ida, verset 48 .

19. Sayyid Qотв, Fi Zilâl al-Qor'ân, Beyrouth, Qotb, Dâr al-Shurûq, 1982. 
tafsîr, du Coran. On voit bien ce qui le distingue d'autres penseurs de mouvements qui se réclament de l'islam. Il n'émet pas de fatwa comme peuvent le faire ceux que l'on appelle les "salafistes". Il ne compose pas un livre de fiqh comme peuvent le faire des oulémas rattachés à une institution religieuse (Mawsu'at $a l$-fiqh) et ne mène pas de réflexion globale sur le fiqh ou la sharî $a$ (par exemple Allal al-Fassi, qui fut un ouléma et l'homme de la lutte pour la libération nationale au Maroc, ou plus récemment A. Raïssouni qui est l'un des principaux penseurs du mouvement réformiste des années 1990 avec son livre sur le Fikr al-maqâsidî̀). C'est donc un livre qui ne tient pas à proprement parler de discours sur les normes juridiques en islam. Alors, à quel type de littérature correspond le minhâaj ? En fait, si on tente de mettre de côté le discours politique d'A. Yassine, on voit bien que al-minhâj an-nabawî est très proche, notamment dans sa seconde partie de la structure des textes soufis, particulièrement de ceux que l'on pourrait appeler le soufisme toléré ou le soufisme légitime aux yeux des tenants de l'islam scripturaire. Je pense en particulier à al-Ghazzâlî et à Abdelkader al-Jilânî qui sont les deux principaux auteurs soufis cités par A. Yassine dans son livre (en particulier Jilânî qu'il cite abondamment). Pourquoi ces deux références ? Parce que al-Ghazzâlî et al-Jilânî sont les références soufies les moins contestées par ceux qui se veulent les tenants de l'orthodoxie.

Intéressons-nous quelques instants à ces deux grandes figures. Al-Ghazzâlî (450-505/1058-1111), qui fut assez tôt un personnage éminent de Bagdad, est sans doute le penseur musulman qui est allé le plus loin dans la tentative de concilier mystique et Loi. Élève du savant Juwayni, al-Ghazzâlî a eu une première formation de juriste. Alternant des périodes de scepticisme et de retraite du monde avec des activités d'enseignement que les souverains (Nizam al-Mulk puis son fils Fakhr al-Mulk) attendaient de lui, il laissa à la postérité une œuvre parmi les plus riches dont le livre phare que cite A. Yassine et qu'il peut être utile d'ouvrir. $L$ a revivification des sciences de la religion, 'Ihyâ' 'ulum ad-dîn se structure en quatre parties : les deux premières traitant essentiellement du fiqh (cultuel, 'ibâdât et social, mu'âmalât), la troisième traite des mublikât (les perditions), la quatrième des munjiyât (les salutaires, par exemple avec un chapitre sur la pauvreté et l'ascétisme kitab al-fiqr wa $z$-zuhd... ${ }^{20}$. Homme de fiqh, al-Ghazzâlî, loin de repousser les normes juridiques tirées par les juristes à partir de leur interprétation du Coran et de la Sunna, espère montrer ce qui a été occulté (mâ uhmila fi fiqh al-'ibâdât) de spirituel dans les normes prescriptives. Mais l'entreprise d'A. Yassine s'en écarte dans la mesure où il va très peu traiter des questions de fiqh. En fait A. Yassine s'appuie sur al-Ghazzâlî en grande partie parce qu'il a la caution de défenseur du sunnisme et de pourfendeur du «batinisme ». Comme le rappelle M. Watt à propos d'un de ses derniers ouvrages: "when he became a mystic he did not cease to be a good Muslim [...], there was no radical change in his

20. Mohammed Al-GhazAli, Ihlya' 'ulum al-din, Le Caire, n.d., 5 tomes. 
theological views, [...] only a change in his interests, and some of his earlier works in the field of dogmatics are quoted with approval in al-Munqidh $»^{21}$.

Dans le même ordre d'idées, al-Jilânîn ${ }^{22}$ (470/1077-561/1166) apporte aussi sa caution de juriste hanabalite. Aussi bien estimé par Ibn Taymiyya, le pourfendeur de l'islam populaire et du culte des saints, qu'adulé par Ibn 'Arabi qui le considérait comme le "pôle des pôles ", al-Jilânî s'est toujours montré attaché au Coran et à la Sunna dans sa pratique du soufisme. Très jeune à la tête d'une madrasa dans la Bagdad abbasside, il a toujours refusé les déviations en matière de soufisme et n'a pas hésité à condamner la Karramiyya et Salimiyya qui rejetaient tous les aspects extérieurs de la religion. Trouvant son inspiration chez Hasan al-Basrî, al-Bistâmî et Junayd, s'inscrivant dans la continuité de al-Ghazzâlî, le dhikr était pour lui tout aussi important que la prière et le jeûne, car le fiqh et le soufisme étaient, dans sa vision des choses, indissociables pour tout croyant qui aime Dieu.

\section{Concilier mystique et prescriptions légales}

Ouvrons un instant le livre d'A. al-Jilânî : Kitâb al-fath ar-rabbânî wa al-fayz al-rahmân $\hat{\imath}^{23}$. Ce livre se compose de soixante-deux majâlis qui sont autant d'étapes qui doivent permettre au croyant de se rapprocher de Dieu dans le long chemin qui l'y conduit. Le texte est écrit sur le mode de la transcription par le disciple des leçons données par le cheikh dans le ribât (couvent) ou à l'école. Aussi ce sont des écrits destinés à être performatifs, à être vécus comme l'indique l'usage de l'impératif et la récurrence dans tout le texte de $\mathrm{Ya}$ Ghulâm! O novice! Ils parlent avant tout au cœur car ce sont des enseignements destinés à être vécus dans l'intériorité du croyant. Lisons un court extrait du livre de al-Jilânî :

O novice, tu n'as pas été créé pour rester dans la vie terrestre et pour y prendre plaisir. Change donc en toi ce que Dieu le Tout-puissant et le Majestueux n'aime pas ${ }^{24}$.

Ce n'est pas un discours informatif ou descriptif ou savant mais bien un discours performatif/impératif. Si le discours d'A. Yassine ne prend pas exactement la même forme, il s'en rapproche néanmoins :

Il est nécessaire pour chaque croyant [...] de commencer sa prière du lien par la Fâtiha puis il doit demander pardon à Dieu pour ses fautes, et il doit demander la miséricorde divine pour la vie terrestre et l'autre vie pour lui-même, pour ses géniteurs et sa famille

21. Montgomry WatT, The Faith and Practive of Al-Ghazali, Londres, G. Allemand Unwin, 1953, p. 12-14.

22. Sur Guilâni (al-Jilânî) on peut consulter Aïni MeHmed Alı, Un grand saint de l'Islam, Guilâni, Paris, Geuthner, 1938 et également, Alain DeEmerseman, Nouveau regard sur la voie spirituelle d'Abdel-Qadir al-Jilani et sa tradition, Paris, Vrin, 1988.

23. Abdelqader JiLANI, Kitâb al-fath al-rabbânî wa al-fayz ar-rahmân̂̂, Le Caire, Boulaq, 1864, (rééd. Paris, Bibliothèque nationale de France).

24. Ibid., 2e majlis, p. 9. 
et il doit saluer le Messager de Dieu et ses Prophètes ainsi que ses Messagers. Puis sur les califes bien guidés, sur les compagnons [du Prophète], et les épouses... ${ }^{25}$

Ce qu'écrit A. Yassine est destiné à être mis en application. C'est un discours performatif bien qu'indirect, puisqu'il n'utilise pas la deuxième personne de l'impératif mais s'adresse au croyant. Cet extrait montre aussi comment des préoccupations mystiques vont de pair avec des obligations cultuelles et porte à conséquence en matière de fiqh des 'ibâdât, de droit cultuel parce qu'il dit clairement les formules à répéter, les hommes à citer, l'ordre dans lequel il convient de le faire... Al-minhâj an-nabawî apparaît donc, au même titre que les tentatives de al-Ghazzâlî et al-Jilânî, comme un livre destiné à ceux qui veulent s'initier à la mystique dans le respect de la Loi.

Chez al-Jilânî, « l'étude du droit en religion est le moyen de connaître le moi », Al-fiqh fî ad-dîn sababun li-ma'rifati an-nafs ${ }^{26}$. A. Yassine entend montrer qu'il souscrit pleinement à ce postulat ${ }^{27}$. Soucieux d'éviter la "sacralisation des hommes pieux» (taqdîs ar-rajul as-sâlih), il souhaite bien prouver au lecteur qu'il entend respecter les normes juridiques de l'islam :

l'amour, le respect et la louange dans les limites de la Loi, fî hudî̀d ash-sharî'a, voilà ce qui convient au hommes pieux de cette Umma, qu'ils soient vivants ou morts. ${ }^{28}$ "

D’ailleurs lorsqu'il cite al-Jilânî, il précise bien que :

Cet homme pieux - que Dieu l'ait en sa miséricorde - n'est pas l'unique témoin de ce secret qu'est le compagnonnage des amis de Dieu. Nous faisons référence à lui justement parce qu'il y a un consensus des oulémas de la Umma sur sa piété, sa guidance et sa vertu ${ }^{29}$.

Souhaitant s'inscrire dans la tradition scripturaire de la Sunna, A. Yassine, tout en construisant son livre sur des équivalents de majâlis soufis de al-Jilânî, utilise à la fin de chaque chapitre ce qu'il appelle les sections de la foi, "shu'ab al-imân » composées de hadîth-s qui sont numérotés de 1 à 77 et qui sont utilisés comme appui aux dix séances. Les hadîth-s se distribuent suivant les dix séances entre le premier hadith qui parle de l'amour de Dieu et de son Prophète (Mahabbat Allah wa rassulih) au dernier hadîth qui parle de l'appel à Dieu le Très-Haut et le Majestueux (Al-da'wa ilâ Allab'azza wa jalla).

25. Abdessalam Yassine, Al-minhâj an-nabawi, p. 130.

26. Abdelkader JiLÂNI, op. cit., p. 129, $39^{\mathrm{e}}$ séance.

27. Dans l'histoire du monde musulman et particulièrement du Maghreb, concilier la Loi et la mystique apparaissait chose beaucoup moins périlleuse qu'elle ne peut sembler aujourd'hui. Jacques Berque écrit notamment à propos du XVII ${ }^{e}$ siècle : "Le mysticisme du temps, combine la tradition la plus savante venue d'Andalousie ou d'Orient avec une poussée rurale ». Le personnage d'Al-Yusi combine "deux courants d'hagiologie populaire et de spéculation savante ", Jacques Berque, Al-Yusi, Paris-La Haye, Mouton et Cie, 1958, p. 121. Voir aussi le livre de Houari Touati, Lettrés, Saints et Sorciers au Maghreb, Paris, Éditions de l'EHESS, 1994.

28. Abdessalam Yassine, op. cit., p. 127.

29. Abdessalam YAssine, op. cit., p. 128. 


\section{Mystique et politique}

Ces efforts de conciliation entre des approches de la religion parfois contraires sont moins déterminants dans la structure du Minhâj que ne l'est la volonté d'A. Yassine de traduire sa mystique en termes politiques. Son projet politique est on ne peut plus explicite:

Le programme d'éducation et d'organisation que nous présentons ne peut s'appliquer que dans deux cas :

si les gouvernants sont cohérents avec leurs principes déclarés de liberté publique, ils accepteront alors l'existence du mouvement islamique reconnu aux côtés des autres mouvements et partis politiques. Et le moins que l'on puisse demander aux gouvernants des musulmans est qu'ils reconnaissent le droit du peuple musulman à exercer l'islam sans directives et oppression du gouvernement;

si les gouvernants reconnaissent leurs torts dans l'oppression des islamistes et s'ils s'engagent à soutenir le mouvement islamiste afin qu'ils se réconcilient avec Dieu et le peuple musulman et s'ils le démontrent en contractant une charte avec Dieu et les croyants qui les engage à gouverner avec ce que Dieu a révélé après une phase transitoire dans laquelle les croyants auront pleine liberté et plein soutien dans la construction d'une entité politique islamique qui préparera des élections islamiques, une constitution islamique et un gouvernement islamique ${ }^{30}$.

Le projet politique d'A. Yassine est largement déterminé par l'idée qu'il se fait du rapport des croyants à Dieu, c'est-à-dire essentiellement le rapport mystique. Non seulement la place du cheikh médiateur entre Dieu et les hommes est indispensable mais la transcendance vécue lors des rites soufis, le sentiment d'élévation et de rapprochement de Dieu doit être un sentiment présent à tous les instants et dans tous les actes des hommes. La structure du livre est révélatrice à cet égard. Consacrant l'essentiel de son ouvrage aux dix séances (khisâl) qui permettront à l'homme de revivifier sa foi, A. Yassine reprend largement les thèmes soufis : al-subba (compagnonnage), al-dhikr (remémoration), al-sidq (la sincérité), al-badl (le don), al-ilm (le savoir), al-jihâd (la lutte contre l'ego).

Mais il faut bien voir que la pensée d'A. Yassine se déploie constamment sur le registre de l'éducation, tarbiyya et de l'organisation, tanzîm. La tension est permanente entre une éducation soufie et une action qui se veut révolutionnaire dans le monde. Prenons l'exemple de la subba, compagnonnage, pour laquelle A.Yassine s'appuie sur les écrits de al-Jilânî :

Et concernant la nécessité d'un cheikh éducateur pour celui qui aspire à arriver à Dieu, et à le connaître et à s'en rapprocher, il [Jilânî] dit : " Deviens l'ami des inspirateurs des cœurs afin que tu aies un cœur. Tu ne peux te passer d'un cheikh sage agissant par les sentences de Dieu le Très-Haut et le Majestueux, qui te donnera le savoir et te conseillera ${ }^{31}$.

Tant qu'il ne traite que de la subba dans le registre de l'éducation, A. Yassine se cantonne à l'initiation mystique menée par un cheikh. En abordant la subba

\footnotetext{
30. Abdessalam Yassine, op. cit., p. 2.
}

31. Abdessalam YAssine, op. cit., p. 128. 
dans le registre de l'organisation, c'est-à-dire en pensant à l'action politique, il transforme le cheikh soufi en chef politique :

dans les sociétés de barbarie et de discorde, de jâhiliya et de fitna, les relations de despotisme sont généralisées à tous les niveaux. En Islam, la da'wa précède la dawla et si la da'wa et la dawla sont réunies dans la main de la jamấa des croyants, alors l'émir éducateur a une fonction d'éducateur qui n'est pas séparée de sa fonction de pouvoir, puisqu'au contraire cette dernière fonction s'appuie sur la précédente et se fortifie par elle, on obéit aux détenteurs de l'autorité légitime parmi nous car Dieu nous a ordonné cela. On ne peut se passer du pacte avec l'émir car c'est un pacte légal mais l'obéissance à l'émir et le bienfait de son compagnonnage, de la considération et de la révélation qu'on lui porte relèvent de la pratique cultuelle ${ }^{32}$.

Cette fusion des procédés mystiques dans l'action politique est généralisée par A. Yassine dans ses dix séances. Même des rites mystiques comme les litanies qui peuvent a priori sembler loin de l'action politique sont repris dans le projet révolutionnaire yassinien. Chez les soufis le $d h i k r$ est remémoration de Dieu, il permet de fortifier la foi en répétant des milliers de fois par jour les formules données par le cheikh. C'est à ce titre le meilleur moyen de se rapprocher de Dieu. A. Yassine cite une nouvelle fois al-Jilânî :

Il [al-Jilânî] a dit, que Dieu lui accorde sa miséricorde, à propos de ceux qui étaient inspirés : “celui dont la foi se fortifie et qui réussit à l'entretenir, voit avec son cœur tout ce dont Dieu l'a informé du jour dernier. Il voit le paradis et l'enfer et ce qu'il y a en eux. Il voit les images et les anges qui l'aident. Il voit les choses telles qu'elles sont. Il voit la vie terrestre et son crépuscule et le retournement de fortune des hommes. Il voit les créatures comme des tombes qui avancent. Et s'il voit au-delà les tombes, il ressentira ce qu'elles comportent de souffrance et de bienveillance divine. Il voit dans le jour dernier la toute-puissance et la bonne fortune. Il voit la miséricorde de Dieu et la souffrance qu'il peut infliger" ${ }^{33}$.

Quelques lignes plus loin, A. Yassine estime que ces moments de $d h i k r$ ne sont pas destinés à « demander la réussite pour soi-même et les honneurs. Au contraire, il s'agit de demander à Dieu qu'il pardonne au croyant, qu'il l'élève dans l'échelle de sa proximité et qu'il lui donne toute la foi et le bel agir ${ }^{34}$ ».

Lorsqu'il en vient, après avoir traité du dhikr en tant qu'éducation, à aborder le $d h i k r$ en tant organisation du mouvement, il transforme une nouvelle fois les exigences mystiques en modes d'action politique : « Le dhikr n'est pas seulement un travail salutaire au niveau des consciences et des mots qui sortent de la bouche et des rites extérieurs pratiqués par le croyant. Le $d h i k r$ signifie aussi se lever dans les mains de Dieu lors de la prière. Les soldats de Dieu le pratiquent pour remplir leur devoir cultuel et parce que c'est un signe de la souveraineté de Dieu dans les relations de Dieu avec ses soldats et dans les relations des soldats de Dieu entre eux. C'est s'apprêter à appliquer la Loi de Dieu le jour où le pouvoir

32. Abdessalam Yassine, op. cit., p. 132.

33. Abdessalam YASSINE, op. cit., p. 148

34. Abdessalam YAssine, op. cit., p. 149. 
reviendra aux croyants, dans tous les domaines du pouvoir, de la politique, de l'économie, de la société, de la justice de la culture et du jihâd".

Pris dans sa forme historique (et notamment chez al-Jilânî), le $d h i k r$ est plutôt la voie du renoncement à la vie dans le monde. Pour Yassine, le $d h i k r$ n'est pas simplement un travail sur soi, dans l'intériorité propre du croyant destiné à se rapprocher de Dieu. C'est aussi un rappel de la société à Dieu à tous les instants, un mode de préparation à l'action sociale et politique afin de permettre aux soldats de Dieu de généraliser ce rappel à Dieu à toute une société oublieuse de Dieu.

\section{Conclusion : un nouveau rapport de sens ?}

Il convient de prendre en compte les ruptures qui rendent possible ce nouveau rapport de sens à la tradition islamique. L'urbanisation de masse, en consacrant l'individualisme et l'anonymat, réduit considérablement le rôle que pouvait jouer autrefois la famille ou la communauté villageoise. La nécessité d'une protection face à la dislocation des liens sociaux traditionnels et face au chaos de la vie urbaine, comparée à celle des communautés villageoises, se fait d'autant plus ressentir que la famille est impuissante à remplir ce rôle. À cela s'ajoute l'émergence de l'État moderne et la montée en puissance du colonialisme européen qui vont, tout en fonctionnarisant les oulémas, contribuer à figer le fiqh, autrefois concept dynamique. L'urbanisation va aussi rendre possible l'apparition de nouveaux groupes sociaux qui vont faire usage des nouveaux moyens de diffusion des discours (livre imprimé, radio, médias) qu'offre l'idéologie politique moderne en vue de conserver le pouvoir ou à l'inverse de le contester ${ }^{35}$. Le développement de ces nouveaux instruments de pouvoir va de pair avec les changements démographiques. De nouvelles générations ${ }^{36}$ qui sont scolarisées dans la ville et dont la famille est généralement issue du monde rural vont faire usage de la lecture et de l'écriture dans leur quotidien et dans leur rapport aux institutions de la vie sociale, économique ou politique ${ }^{37}$. Ces changements vont par conséquent

35. Comme l'écrit B. Messick « Once the central discourse of the Muslim polity, the shari'a was decisively repositioned within nation-state frameworks. My characterization of the shari'a as a genereal societal discourse rather than as Islamic law placed emphasis on a historical transition to the codified and legislated form of law. The decisive move from the old manuals to legislation, form open to closed shari'a texts represents both the key instance of discursive transformation and an important backdrop for changes that have occurred in other institutions. Alteration in the form of the shari'a has changed the nature of not only of interpretation, but also of government ». Brinkley MEssick, The Calligraphic State, Berkeley, University of California Press, 1993, p. 253. 1989.

36. Sur cette question cf. Karl Mannheim, Le Problème des générations, Paris, Nathan,

37. Sur les conséquences sociologiques de l'éducation moderne dans le cas du Maroc voir ce que Dale Eickelman appelle "The great transformation ", Dale EICKELMAN, Knowledge and power in Morocco, Princeton, Princeton University Press, 1985, p. 161. 
introduire de nouvelles manières d'aborder le rapport à la religion car ces nouvelles générations ne peuvent plus se satisfaire de l'islam rural ${ }^{38}$ qui devient souvent à leurs yeux trop fruste ou pas assez universel ${ }^{39}$. Ces nouvelles générations ont d'autant plus besoin d'une médiation d'un nouveau type entre Dieu et les hommes que l'extension du champ d'application des normes «les rend moins applicables à des contextes réels. En d'autres termes il devient difficile et peutêtre impossible à un individu ou à un groupe de vivre conformément à ce type de morale universaliste ou d'injonction éthique " ${ }^{40}$. Ces groupes sociaux ont, en définitive, besoin d'un discours et d'une pratique qui puissent répondre à leur vision du monde, à leurs besoins et à leur mode de vie, c'est-à-dire un discours et une pratique dans lesquels ils peuvent se reconnaître et qu'ils peuvent s'approprier $^{41}$.

Youssef BELAL

Institut d'Études Politiques de Paris

38. Abdellah Hammoudi note dans son étude sur le sacrifice au Maroc que les jeunes éduqués dans les villes considèrent désormais certaines fêtes de leur village comme bid'a, innovation blâmable, cf. Abdellah Hammoud, La victime et ses masques, Paris, Seuil, 1988.

39. Jack Goody : "Les systèmes étatiques ont inévitablement tendance à appliquer des normes à l'échelle de l'État, du moins dans les sphères importantes [...]. Si la religion est liée à quelque niveau que ce soit au système politique, alors un gouvernement centralisé aura tendance à avoir un effet similaire sur certains éléments du culte. Les codes écrits portent ce processus de généralisation, de consolidation à un degré supérieur ». D’autre part, "Dans les codes écrits, il existe une tendance à présenter une seule formule abstraite qui recouvre, et dans une certaine mesure remplace les normes plus contextualisées des sociétés orales ». Jack Goody, La logique de l'écriture, Paris, Armand Colin, 1986, p. 24-25.

40. Ibid., p. 27.

41. Dans le cas de l'Égypte, Michael Gilsenan montre bien comment les confréries soufies ne répondent plus aux besoins de nouvelles catégories sociales. Il fait un parallèle entre le déclin des turuq (voies), et l'ascension du mouvement des Frères Musulmans : "All this it did in the name of Islam, but of an Islam in which the stress was very different from the passive retreatism of the Orders. The Brotherhood directed its followers to the working out of God's purpose in the world. It offered a vision of the future cast in religious terms. But though these terms derived their meaning and affective symbolic connotations from the past, they were made to encompass the material world of the present. They were to guide positive social action on Islamic principles interpreted as a key to the reordering of a society dislocated by change. Individuals had status according to their efforts in the holy struggle, and not according to the uncertain new criteria which had appeared in the evolving pattern of social differentiation ", Michael GILSENAN, Saint and Sufi in Modern Egypt, Oxford, Oxford University Press, 1973, p. 204. 
I84 - ARCHIVES DE SCIENCES SOCIALES DES RELIGIONS

\section{Résumé}

Au Maroc, le mouvement islamique et mystique "Justice et bienfaisance " est étroitement lié à la personne de son fondateur Abdessalam Yassine. L'idée principale développée ici est que Abdessalam Yassine est à la fois médiateur religieux et médiateur politique. Etre sous son autorité spirituelle signifie pour lui et ses adeptes être sous son autorité politique. Cet article tente de comprendre les ressorts de cette synthèse entre religion et politique en étudiant une ziyâra [visite] au maître à Salé, et quelques passages du livre le plus important de Abdessalam Yassine dans lequel il s'appuie sur un soufisme scripturaire.

Mots-clés : mouvement islamique, mystique, politique, autorité spirituelle.

\section{Abstract}

In Morocco, the mystical Islamic movement "Justice and Beneficence" is strongly linked to the personality of his founder, Abdessalam Yassine. The argument is that Abdessalam Yassine is a religious mediator and a political mediator at the same time. Being under his spiritual authority means for him and his enthusiasts being under his political authority. In order to demonstrate this hypothesis two main points are developed. First, this article analyses a ziyâra, a visit to the master at Salé. Second, it deals with some passages of his most important book in which Abdessalam Yassine relies on a scriptural Sufism.

Key words: Islamic movement, mystic, politics, spiritual authority.

\section{Resumen}

En Marruecos, el movimiento mistico islamico «Justicia y beneficencia » es estrechamente atado a la persona de su fundador Abdessalam Yassine. La idea principal desarollada aqui es que Abdessalam Yassine es a la vez un mediador religioso y un mediador politico. Estar bajo su autoridad espiritual significa, por él y sus seguidores, estar bajo su autoridad politica. Ese articulo trata de demostrar esa hipotesis, analizando una ziyâra, visita al maestro en Salé, y algunos pasajes de su libro el mas importante en el cual Abdessalam Yassine se basa en un sufismo escripturario.

Palabras claves : movimiento islamico, mistica, autoridad politica, autoridad esperitual. 